\title{
The genetic status of the introduced giraffe population in Central South Africa
}

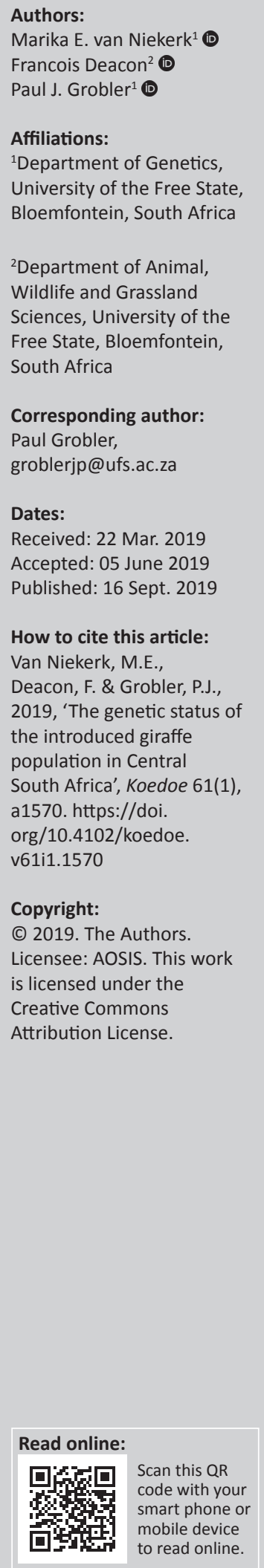

\section{Introduction}

There has been no reliable historical evidence of giraffe occurring naturally in the Free State Province in Central South Africa (Dagg 1962; Deacon \& Parker 2016; Sydney 1965). Although Lynch (1983) mentioned the possibility of the natural distribution of giraffe in the East and Western Free State, there is no concrete evidence that giraffe existed earlier than this in the Free State (Ansell 1968). According to Griesel (1961), Hirst (1966), Lambrechts (1974) and Terblanche and Kok (1995), translocations of the species into protected areas and private land in the Free State Province happened in any case, sometimes regardless of the natural habitat preferences of giraffe (Castley, Boshof \& Kerley 2001; Deacon \& Tutchings 2019). These authors also affirmed that giraffe had, however, occurred naturally westwards of the Free State, in the Northern Cape Province. The introduced status of the giraffe population in Central South Africa, and in particular the Free State Province, thus raises questions regarding the origin and the taxonomic status of these animals.

Although giraffe is an introduced species in the Free State Province, it is also a reality that extralimital populations of large mammals add value to ecotourism in self-financing private protected areas (Maciejewski \& Kerley 2014) and are therefore kept on many properties. Extralimital animals can also still add conservation value by contributing to long-term population survival, while extralimital populations could in some cases nevertheless be a reservoir of valuable genetic resources. The newly created ownership in wildlife species not only contributes to diversity in wildlife species for ecotourism, but also assists to protect valuable and key resource areas and habitat for all fauna and flora.

Giraffe taxonomy and subspecies status have been the subject of much debate over the last century. It should also be noted that there have been only limited representation from South African populations in the reported studies. Bercovitch and Deacon $(2015,2017)$ stated that there are currently at least four different taxonomic classifications for giraffe with the current global acceptance of nine subspecies (Muller et al. 2016). Most recently, Fennessy et al. (2016) and Winter et al. (2018) suggested a new taxonomy for giraffe with four genetically distinct giraffe species instead of one, but this has not been accepted by the International Union for Conservation of Nature (IUCN) who requested more evidence and a larger sample size; and the status quo at least for now is one species with nine subspecies (Bercovitch et al. 2017). It should also be noted that the aforementioned genetic studies have only limited representation from South African populations. Irrespective of the exact classification system used (one or four species), all the current classification systems include recognition of a 'South African giraffe' (Giraffa camelopardalis giraffe/G. giraffa giraffa) and the 'Angolan giraffe' (Giraffa camelopardalis angolensis [G. c. angolensis]/Giraffa giraffa angolensis [G. g. angolensis]). Fennessy et al. (2016) suggested that South Africa harbours only the South African giraffe. However, Deacon and Parker (2016) state that the Angolan giraffe has been translocated from Namibia to countries such as Botswana and South Africa, and some game ranches might contain a mixture of subspecies, or even hybrid giraffe. The extent of historical natural migration of animals between regions is also not known. The available data on historical and current distribution ranges of South African giraffe are shown in Figure 1 (based on the IUCN Red List assessment of Muller et al. 2018).

In addition to taxonomic considerations, genetic diversity within giraffe populations in the region is also a potential cause for concern (Deacon \& Tutchings 2019). In the Free State Province, giraffe are almost invariably found in small populations, for reasons of habitat availability or because they may not be utilised as intensively as some other wildlife species. The number of giraffe found on farms thus ranges from populations numbering 10-30 down to populations with single pairs of animals, with the potential risk of decreased genetic diversity and ultimately reduced 


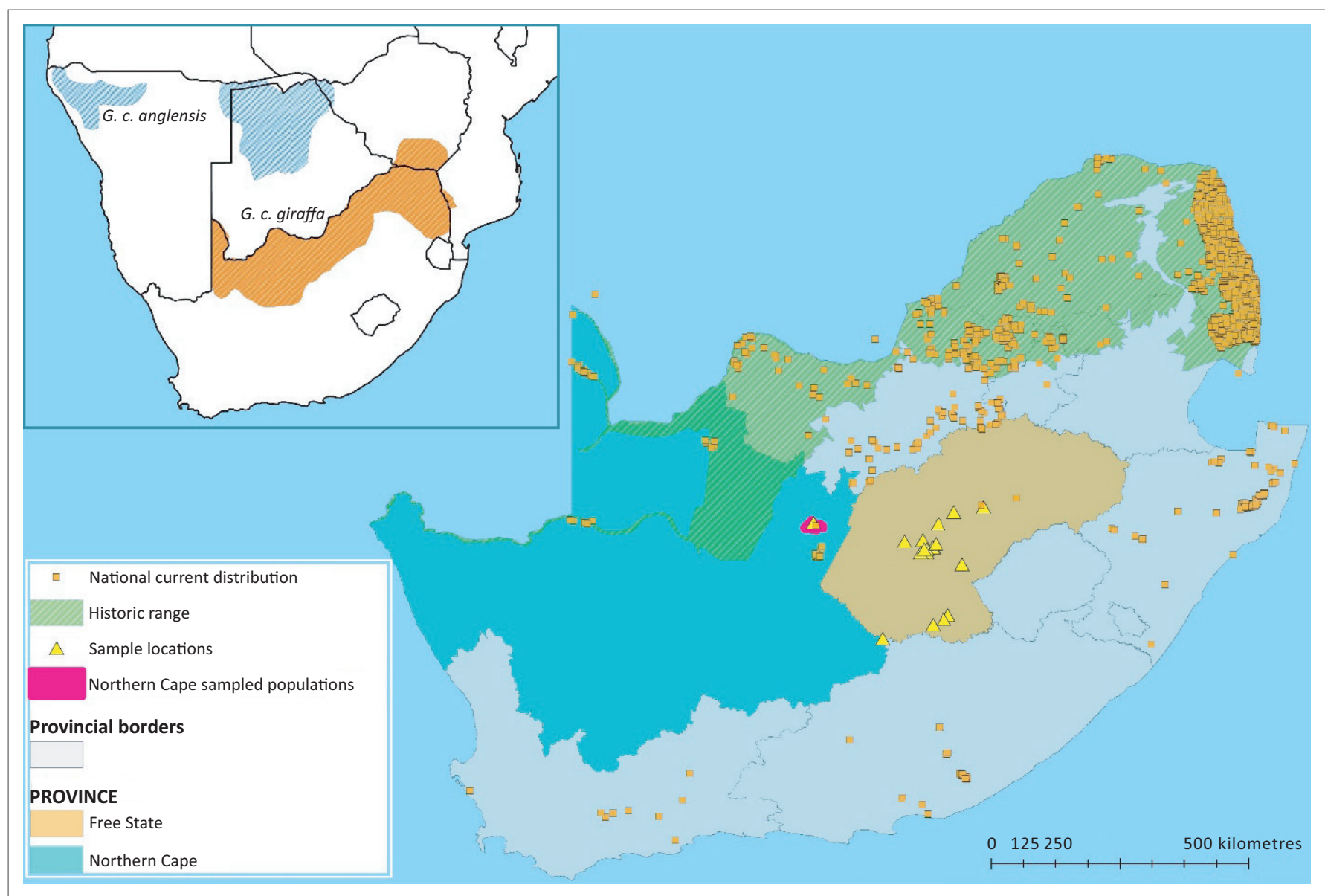

Source: Adapted from Deacon, F. \& Tutchings, A., 2019, 'The South African giraffe Giraffa camelopardalis giraffa: A conservation success story', Oryx 53(1), 45-48. https://doi.org/10.1017/ S0030605317001612

FIGURE 1:The current and historical distribution of giraffe in South Africa, regions sampled and localities referred to in the text.

fitness or adaptability. Management strategies to maintain diversity is needed, but, conversely, the necessary translocations needed to conserve genetic diversity could hold risks if the taxa involved are significantly different, in line with theory on the mixing of distinct taxonomic units (Crandall et al. 2000; Moritz 2002) and outbreeding depression (Edmands 2006).

With little being known about the effect of extralimital introductions and fragmentation in giraffe populations in Central South Africa, the aim of the current project was to determine the genetic structure of giraffe in Central South Africa relative to the wider southern African population, and also obtain an estimate of genetic diversity within and between individual giraffe populations.

\section{Methods}

\section{Study sites and sample collection}

We collected samples from three groups: (1) A total of 34 giraffe were sampled across 10 privately owned herds in the Free State Province (FS-Private). The census population size $(N)$ of these groups ranged from 2 to $30+$; and the ultimate origins of the animals in these populations are unknown other than the fact that founding involved several sources and that translocations among populations have occurred in recent times. (2) As representative of giraffe in public protected herds from the Province, nine giraffe were sampled from the Willem Pretorius Provincial Nature Reserve $(N=21)$. Two groups of founders sourced from the Limpopo Province and one group from the KwaZulu-Natal Province were originally used to start this population (FS-Protected area). (3) Finally, four giraffe were sampled from a privately owned herd in the adjacent Northern Cape Province $(N=20)$. Eight individuals were translocated to this locality from Namibia in 1971, with another six individuals translocated from the then Transvaal Province in northern-eastern South Africa in 1973.

We used dung as the preferred sample type, to minimise stress on the individuals, as well as for cost purposes, ethical considerations and ease of access to populations. The procedure followed at each locality was as follows: once a giraffe was found, it was observed until defecation had occurred. The exact spot was noted; the giraffe was characterised using size, sex and patterns on its body to prevent duplicate sampling; and the sample retrieved. Samples were stored in $96 \%$ ethanol and at $-20^{\circ} \mathrm{C}$ as soon as possible after collection. A small number of skeletal muscle and blood samples available opportunistically for specific localities were also included. 


\section{Genetic analysis}

Deoxyribonucleic acid (DNA) was extracted using the Zymo Research ZR Fecal DNA MiniPrep ${ }^{\mathrm{TM}}$ kit used for dung samples, the Zymo Research Quick-DNA ${ }^{\mathrm{TM}}$ FFPE MiniPrep kit for tissue samples and the Roche High Pure PCR Template Preparation kit used for blood samples. A NanoDrop ${ }^{\circledR}$ ND-1000 Spectrophotometer was used to determine the quantity and quality of extracted DNA.

We sequenced sections of the cytochrome $b(C y t b)$ and displacement loop (D-loop) mitochondrial DNA (mtDNA) regions. The $C y t b$ primers used were from a study done by Bock et al. (2014): forward - GTG GAA GGC GAA GAA TCG; reverse - GAA AAA CCA TCG TTG TCG T; with the sequences of primers used for the D-loop region sourced from a study done by Seymour (2001): forward - CCC AAA GCT GAA GTT CTA TT; reverse - CAA TAA CTG TAT GTA CTA TG-3'. PCR reactions were based on the Kapa2 $\mathrm{G}^{\mathrm{TM}}$ Robust HotStart ReadyMix PCR Kit for the Cytb region, with the Ampliqon TEMPase Hot Master Mix A Kit used for the $D$-loop region. Annealing temperatures were $62{ }^{\circ} \mathrm{C}$ and $50{ }^{\circ} \mathrm{C}$, respectively. Polymerase chain reaction (PCR) products were cleaned using the Biospin PCR Purification Kit (Bio-Rad), followed by sequencing (in both directions) using the ABI PRISM ${ }^{\circledR}$ BigDye ${ }^{\circledR}$ Terminator version 3.1 Cycle Sequencing Kit and standard conditions. Products were cleaned using the ZR DNA Sequencing Clean-up ${ }^{\mathrm{TM}}$ Kit, before running on either an ABI3130 or 3500 Genetic Analyser.

\section{Data analysis}

Geneious Pro version 4.7.4 software (Kearse et al. 2012) was used to view, assemble and align all sequences. Mega v7.0.26 software (Kumar, Stecher \& Tamura 2016) was utilised to identify the best model of substitution for each mtDNA region separately. The haplotypes for $C y t b$ and the $D$-loop region were then combined into a concatenated dataset for all subsequent analyses. Phylogenetic trees were constructed using a maximum likelihood (ML) approach, with 1000 bootstrap replications (in MEGA software). To complement the phylogenetic analysis, Network version 5.0.1.1 software (Fluxus Engineering 2019, www.fluxux-engineering.com) was used to construct haplotype networks, using a median-joining approach. Genetic divergence between populations was quantified as the average number of nucleotide differences between populations $\left(D_{x y}\right)$, from DNA Sequence Polymorphism (DnaSP) version 5 (Rozas et al. 2003), and genetic diversity within each group using haplotype diversity $(\mathrm{Hd})$ and nucleotide diversity $(\pi)$ from DnaSP software.

As a reference database for G. c. giraffa and G. c. angolensis, the concatenated mtDNA D-loop and Cytb sequences used by Winter et al. (2018) were made available by these authors. The latter authors also provided sequences for G. c. peralta and G. c. tippelskilchi, to use as an out-group.

\section{Ethical considerations}

The methods employed during this study were approved by the Interfaculty Animal Ethics Committee of the University of the Free State (Numbers: UFS-AED2015/0050 and UFS-AED2015/0066). Collection of samples was sanctioned under permits issued by DESTEA (Permit number: 01/30305) and the Northern Cape Department of Environment and Nature Conservation (Permit number: FAUNA 0729/2017 and FAUNA 0730/2017). Section 20 veterinary approval for the collection, transport and storage of samples was obtained from the National Department of Agriculture, Forestry and Fisheries (DAFF), with approval number 12/11/1/4.

\section{Results}

After alignment of sequences and trimming to equal length, a sequence length of 405 base pairs (bp) was used for further analysis of the Cytb gene, with 275 bp available for the D-loop region. The two $C y t b$ haplotypes observed have been submitted to the GenBank database as MH033837 and $\mathrm{MH} 033838$, and the 10 haplotypes for the D-loop region as $\mathrm{MH} 033839$ to $\mathrm{MH} 033848$. Our concatenated sequences were aligned with the reference sequences (total $1556 \mathrm{bp}$ and all sequences trimmed to the shared $680 \mathrm{bp}$.

The data for the two mtDNA regions were tested separately to determine the model of substitution, and both regions were best described by a Tamura 3-parameter model of substitution. A phylogenetic tree, based on the concatenated dataset and an ML approach, is presented in Figure 2a. All the G. c. giraffa reference sequences form a clade with $75 \%$ bootstrap support, separate from G. c. angolensis. This cluster also contains all the giraffe samples from the Free State protected area and 14 giraffe samples from private farms in the Free State Province. All other giraffe samples from the private farms in the Free State Province, comprising 21 individuals, cluster with G. c. angolensis, as do the four samples from the Northern Cape. The reference G. c. peralta and G. c. tippelskirchi haplotypes formed a distinct cluster with $100 \%$ bootstrap support. The trend from the ML tree is confirmed in the haplotype network presented in Figure $2 b$. Haplotypes of G. c. angolensis and G. c. giraffa form distinct clusters, with giraffe from a Free State protected area clustering with G. c. giraffa and with giraffe from private populations grouping with one or the other subspecies.

The average number of nucleotide differences between reference sample sets of G. c. giraffa and G. c. angolensis was $D_{x y}=0.033$. This value was regarded as representative of the difference between pure populations of the two subspecies and was therefore used as a yardstick to gauge the relative magnitude of values obtained for pairwise comparisons between each of the studied populations and the two subspecies. The population from the Free State protected area showed comparatively low differentiation from G. c. giraffa, with $D_{x y}=0.014$, but with $D_{x y}=0.020$ when compared with G. c. angolensis. The Northern Cape population showed close 


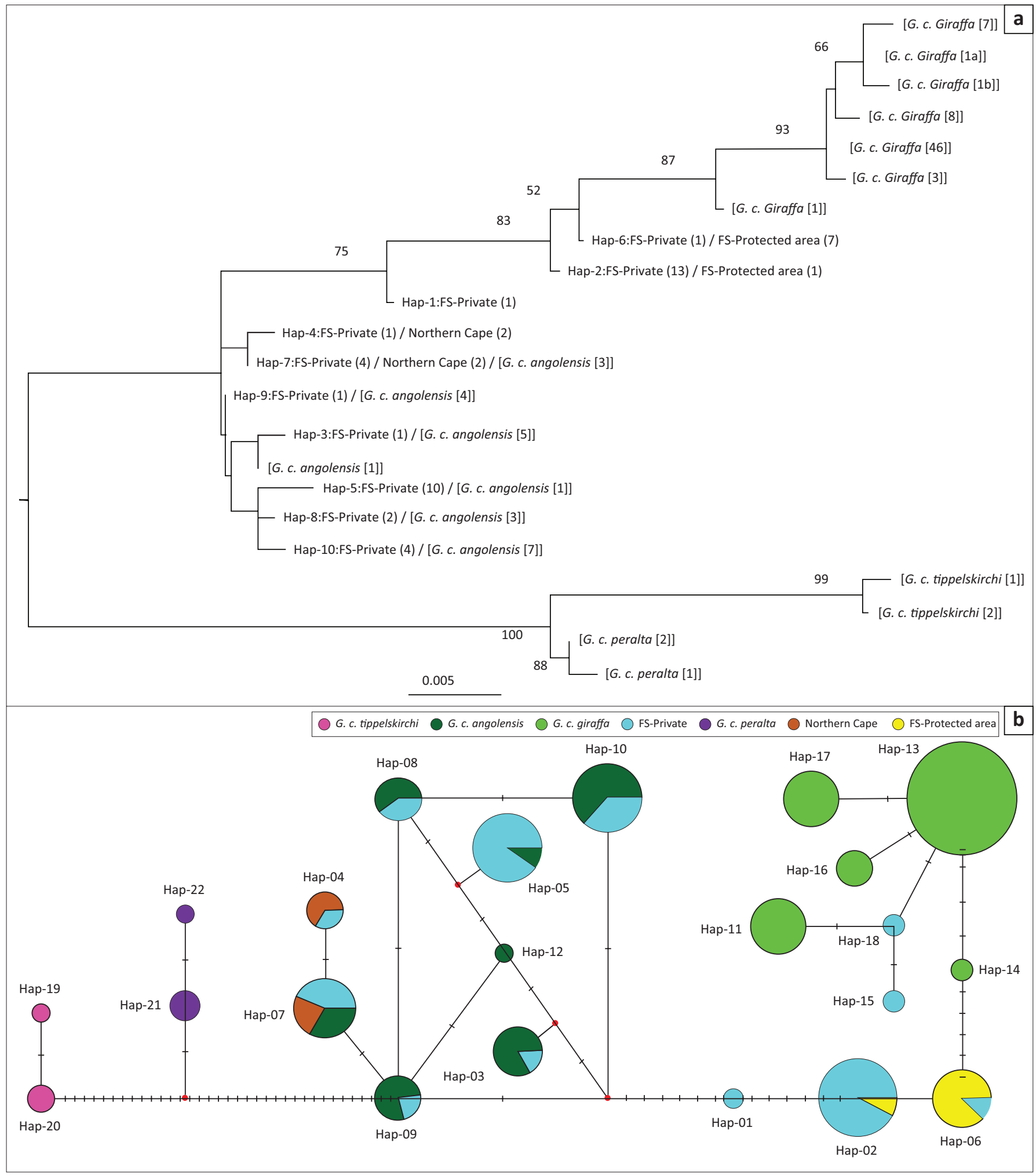

G. c. tippelskirchi, Giraffa camelopardalis tippelskirchii; G. c. angolensis, Giraffa camelopardalis angolensis; G. c. giraffa, Giraffa camelopardalis giraffe; G. c. peralta, Giraffa camelopardalis peralta; FS-Private, Free State Private; FS-Protected area, Free State protected area; Hap, haplotype.

FIGURE 2: Status of giraffe from Central South Africa, relative to reference giraffe populations, based on a concatenated mitochondrial DNA displacement loop (D-loop) and cytochrome $b$ (Cytb) sequence dataset. (a) Phylogeny from maximum likelihood topology of haplotypes. The haplotype names from giraffe analysed for the current study are underlined, with reference samples indicated in [square brackets]. Sample sizes are also indicated. Numbers above branches indicate the percentage of bootstrap replicates where the clade was found. (b) Minimum spanning network. Nodes represent haplotypes, with the size of each node showing the number of individuals that share that haplotype. Crossbars reflect the number of mutational events between specific haplotype pairs.

identity with G. c. angolensis, with $D_{x y}=0.004$, but $D_{x y}$ between the Northern Cape group and G. c. giraffa was much higher at 0.034, thus equalling the distance between G. c. giraffa and
G. c. angolensis. The $D_{x y}$ value between the privately owned giraffes and G. c. giraffa and G. c. angolensis was 0.028 and 0.009 , respectively. 
Levels of genetic diversity within groups were as follows (with format - number of haplotypes/number of polymorphic sites/haplotype diversity [Hd] and nucleotide diversity $[\pi]$ : FS-private populations - 10/19/0.814/0.011; FS-protected area $-2 / 1 / 0.389 / 0.001$ and Northern Cape-2/1/0.667/0.008. By comparison, values for the reference datasets were G. c. giraffa - 7/6/0.508/0.001 and G. c. angolensis $7 / 6 / 0.844 / \pi=0.003$.

\section{Discussion}

Although we were not able to sequence the full length of mtDNA used by Winter et al. (2018), results from the current study provided valuable new data on the status of the giraffe population in Central South Africa. Based on the concatenated mtDNA Cytb and D-loop dataset used, a total of 23 individuals classified as G. c. giraffa. These animals originated from a Provincial Nature Reserve and six private game farms. This is in line with expectations that locally sampled individuals would have mtDNA lineages of what is currently regarded as the South African giraffe subspecies. Unexpectedly, 24 individuals from the present study classified as G. c. angolensis. This outcome suggests that a significant number of individuals with mtDNA lineages of the Angolan giraffe rather than the South African giraffe are present in the Central South African giraffe population. We note that those individuals displaying G. c. angolensis haplotypes may also be hybrids, either first generation or more advanced generations, resulting from initial crosses involving G. c. giraffa males and G. c. angolensis females. (Conversely, some of the individuals classified as G. c. giraffa may also be hybrids, with male G. c. angolensis individuals in their pedigrees.) Population-genetic analysis based on the average number of nucleotide differences between groups confirmed that the overall privately owned giraffe herds in Central South Africa show an unexpected identity with G. c. angolensis.

There are two possible scenarios to explain the observation of a G. c. angolensis lineage in South African populations, based on artificial translocations and unrecorded historical migrations. The giraffe is a popular game ranching species, and there has been substantial commercial movement of the animal around the sub-continent (Deacon \& Parker 2016). The findings that some of the giraffe sampled in the Free State are potentially Angolan giraffe may exemplify how the subspecies has been given a more southern distribution to what was previously thought, through translocations. This can be partly explained by the close historical and current link between South Africa and Namibia, with substantial translocation of game animals between the two countries, and individuals of G. c. angolensis may have found their way into South Africa along this route. For example, the Kglagadi Transfrontier Park contains both introduced G. c. angolensis and G. c. giraffa (Deacon \& Tutchings 2019). We note that game breeders may not be aware of the subspecies status of the giraffe on their properties because this is not currently a publicised conservation concern, and rather focus on maintaining viable populations through introductions from readily available sources.

In an alternative scenario, the current results may suggest that a re-evaluation of subspecies distribution in the region is necessary. In this scenario, G. c. angolensis detected in South Africa may in fact be part of a natural continuum of diversity of lineages existing within and between the two subspecies. Winter et al. (2018) discuss how the historically assumed distribution of the subspecies ranged, and that the current distribution of G. c. angolensis ranges over a wider span than what was previously thought. Bock et al. (2014) found several deviations from expected subspecies distribution. For example, two giraffe that had been assumed to be South African giraffe grouped with Angolan. A large number of giraffe sampled were from the Chobe National Park, Nxai Pans, Vumbura Concession and Moremi Game Reserve in northern Botswana, and Bwabwata National Park in northeastern Namibia. Namibian localities assumed to have G. c. angolensis grouped with G. c. giraffa from the Khamab Kalahari Reserve in South Africa. These authors state that the assignment of giraffe to the incorrect subspecies could be because of either natural migration or human-induced translocation. In their study, Bock et al. (2014) also found that the South African giraffe were also distributed further north than the previously assumed range. Furthermore, Winter et al. (2018) found that the Angolan giraffe had a more eastern distribution than the previously known distribution. There is thus a need for a finer grained study of natural populations, to determine the true borders of subspecies and lineages, and to better understand the spectrum of pure genotypes for both the South African and Angolan giraffe. Within the South African giraffe population, differences between historic origin populations (Kruger National Park) in comparison to translocated populations such as the individuals in Central South Africa should be investigated.

The observed levels of genetic diversity supported our conclusion on the taxonomic status of the groups studied. The population from a public protected area showed least diversity of all groups studied, despite being a sizeable population in the context of this study. However, this population also showed close identity with G. c. giraffa, which has lower diversity compared to G. c. angolensis. Conversely, a small sample of animals from the Northern Cape showed high diversity and also showed close identity to G. c. angolensis, which has higher diversity compared to G. c. giraffa. The highest level of genetic diversity recorded in this study - higher than values estimated for either pure G. c. giraffa or G. c. angolensis - was found in the pooled sample set for privately owned giraffe in the Free State Province. Grobler et al. (2018) showed that hybridisation may result in artificially elevated levels of genetic diversity. The high extant levels of diversity found in the privately owned herds may thus reflect a mix of haplotypes of both G. c. giraffa and G. c. angolensis. 
In addition to providing new data on subspecies status, the results from the current study have significant implications for the conservation of genetic diversity in small giraffe populations in Central South Africa. Some of the individual populations sampled consisted of four or fewer giraffe, sometimes founded using animals bought from a single source population and with all animals bought at the same time. These, and many similar small giraffe populations in Central South Africa, cannot reasonably be expected to add significant numbers of additional giraffe to their populations because of the size of the areas and habitat availability (carrying capacity). Exchange of animals among populations may thus have significant advantages in inducing gene flow. However, this suggested management approach is based on the premise that uncontrolled exchanges are permissible from a taxonomic point of view, that is, that all extant animals classify to the same subspecies or another appropriate unit for conservation. The latter requirement is, however, clearly not met by the extant Free State giraffe populations, based on the trends reported in the preceding sections.

The data presented here provide evidence that both subspecies from southern Africa are present in populations sampled from Central South Africa. This could reflect either translocations or the fact that the borders of the subspecies' ranges are not clearly defined. For future study, we suggest that sampling and analysis be expanded to provide further data on subspecies' distribution and possible admixture. In this regard, museum samples could also be used to elucidate the range of each mtDNA haplotype, and microsatellite makers will provide more precise data on admixture, especially where male mtDNA is not detected in hybrids. Finally, the adaptive significance of genetic differences between subspecies should be investigated.

\section{Acknowledgements}

The authors thank all private landowners and the Free State Department of Economic, Small Business Development, Tourism and Environmental Affairs (DESTEA) for permission to sample populations. The authors thank two anonymous reviewers for their constructive comments that improved the article.

\section{Competing interests}

The authors declare that they have no financial or personal relationships that may have inappropriately influenced them in writing this article.

\section{Authors' contributions}

F.D. and P.J.G. conceptualised the study. M.E.v.N. conducted fieldwork and performed all laboratory analysis. P.J.G. and M.E.v.N. conducted statistical analysis. All the authors contributed to writing this article.

\section{Funding information}

The study was partly financed through the National Research Foundation incentive funding to P.J.G.

\section{Data availability statement}

Sequences generated for this study are available from GenBank.

\section{Disclaimer}

The views and opinions expressed in this article are the authors' own and not an official position of the institution or funder.

\section{References}

Ansell, W.F.H., 1968, 'Part 15. Order Artiodactyla', in J. Meester \& H.W. Setzer (eds.), The mammals of Africa: An identification manual, pp. 1-84, Smithsonian Institution Press, Washington, DC, https://doi.org/10.1111/j.1096-3642.1965. tb00081.x

Bercovitch, F.B., Berry, P.S.M., Dagg, A., Deacon, F., Doherty, J.B., Lee, D. et al., 2017 'How many giraffe species exist?' Current Biology 27, R123-R138, https:// doi:10.1016/j.cub.2016.12.039

Bercovitch, F.B. \& Deacon, F., 2015, 'Gazing at a giraffe gyroscope: Where are we going?' African Journal of Ecology 53, 135-146. https://doi.org/10.1111/aje.12222

Bock, F., Fennessy, J., Bidon, T., Tutchings, A., Marais, A., Deacon, F. et al., 2014 'Mitochondrial sequences reveal a clear separation between Angolan and South African giraffe along a cryptic rift valley', BMC Evolutionary Biology 14, 219. https://doi.org/10.1186/s12862-014-0219-7

Castley, J.G., Boshof, F, A.F. \& Kerley, G.I.H., 2001, 'Compromising South Africa's natural biodiversity - Inappropriate herbivore introductions', South African Journal of Science 97, 334-348. https://hdl.handle.net/10520/EJC97379

Crandall, K.A., Bininda-Edmonds, O.R.P., Mace, G.M. \& Wayne, R.K., 2000, 'Considering evolutionary processes in conservation biology: An alternative to "evolutionarily significant units"', Trends in Ecology and Evolution 15, 290-295. https://doi.org/ 10.1016/S0169-5347001876-0

Dagg, A.I., 1962, 'The distribution of the giraffe in Africa', Mammalia 26, 497-505. https://doi.org/10.1515/mamm-1962-0405

Deacon, F. \& Parker, D., 2016, 'A conservation assessment of Giraffa camelopardalis giraffa', in M.F. Child, L. Roxburgh, E. Do Linh San, D. Raimondo \& H.T. DaviesMostert (eds.), The red list of mammals of South Africa, Swaziland and Lesotho, pp. 1-9, South African National Biodiversity Institute and Endangered Wildlife Trust, South Africa.

Deacon, F. \& Tutchings, A., 2019, 'The South African giraffe Giraffa camelopardalis giraffa: A conservation success story', Oryx 53(1), 45-48. https://doi.org/10.1017/ S0030605317001612

Edmands, S., 2006, 'Between a rock and a hard place: Evaluating the relative risks of inbreeding and outbreeding for conservation and management', Molecular Ecology 16, 463-475. https://doi.org/10.1111/j.1365-294X.2006.03148.x

Fennessy, J., Bidon, T., Reuss, F., Kumar, V., Elkan, P., Nilsson, M.A. et al., 2016, 'Multilocus analyses reveal four giraffe species instead of one', Current Biology 26(18), 2543-2549. https://doi:10.1016/j.cub.2016.07.036

Griesel, J., 1961, 'A new game sanctuary for the Orange Free State, The Willem Pretorius Game Reserve', African Wildlife 15(2), 121-125.

Grobler, J.P., Van Wyk, A.M., Dalton, D.L., Jansen Van Vurren, B. \& Kotze, A., 2018, 'Assessing introgressive hybridization between Blue wildebeest (Connochaetes taurinus) and Black wildebeest (Connochaetes gnou) from South Africa', Conservation Genetics 19, 981-993. https://doi.org/10.1007/s10592-018-1071-x

Hirst, S.M., 1966, Immobilization of the Transvaal giraffe Giraffa camelopardalis giraffa using an oripavin derivative', Journal of the South African Veterinary Association 37, 85 .

Kearse, M., Moir, R., Wilson, A., Stones-Havas, S., Cheung, M., Sturrock, S. et al., 2012 'Geneious Basic: An integrated and extendable desktop software platform for the organization and analysis of sequence data', Bioinformatics 28(12), 1647-1649. https://doi.org/10.1093/bioinformatics/bts199

Kumar, S., Stecher, G. \& Tamura, K., 2016, 'MEGA7: Molecular evolutionary genetics analysis version 7.0.', Molecular Biology and Evolution 33(7), 1870-1874. https:// doi.org/10.1093/molbev/msw054

Lambrechts, A.W., 1974, 'The numerical status of sixteen game species in the Transvaal, excluding the Kruger National Park', Journal of the South African Wildlife Management Association 4, 95-102. https://hdl.handle.net/10520/AJA03794369

Lynch, C.D., 1983, 'The mammals of the Orange Free State', Memoirs van die Nasionale Museum Bloemfontein 18, 1-218.

Maciejewski, K. \& Kerley, G.I.H., 2014, 'Understanding tourists' preference for mammal species in private protected areas: Is there a case for extralimital species for ecotourism?', PLoS One 9(2), e88192. https://doi.org/10.1371/journal pone.0088192 
Moritz, C., 2002, 'Strategies to protect biological diversity and the evolutionary processes that sustain it', Systematic Biology 51, 238-254. https://doi:10.1080/ 10635150252899752

Muller, Z., Bercovitch, F., Brand, R., Brown, D., Brown, M., Bolger, D. et al., 2018 Giraffa amelopardalis (amended version of 2016 assessment), The IUCN Red List of Threatened Species 2018: e.T9194A136266699, viewed 28 June 2018 from https://www.iucnredlist.org/species/9194/136266699n.

Muller, Z., Bercovitch, F., Fennessy, J., Brown, D., Brand, R., Brown, M. et al., 2016 Giraffa camelopardalis, The IUCN Red List of Threatened Species 2016: e. T9194A51140239, viewed 08 December 2016 from www.iucnredlist.org.

Rozas, J., Sánchez-Delbarrio, J.C., Messeguer, X. \& Rozas, R., 2003, 'DnaSP, DNA polymorphism analyses by the coalescent and other methods', Bioinformatics 19 2496-2497. https://doi.org/0.1093/bioinformatics/btg359
Seymour, R.S., 2001, 'Patterns of subspecies diversity in the giraffe, Giraffa camelopardalis (L. 1758): Comparison of systematic methods and their implications for conservation policy', PhD thesis, University of Kent, Canterbury. https://ethos. bl.uk/OrderDetails.do?uin=uk. bl.ethos. 275008

Sydney, J., 1965, 'The past and present distribution of some African ungulates', Proceeding of the Zoological Society of London 30, 139-167. https://doi.org/ $10.1017 /$ S0030605300006815

Terblanche, H.J. \& Kok, O.B., 1995, 'Die voorkoms van wild in die Oranje-Vrystaat', Navorsinge van die Nasionale Museum Bloemfontein 11(4), 61-100. https://hdl. handle.net/10520/AJA00679208_1465

Winter, S., Fennessy, J., Fennessy, S. \& Janke, A., 2018, 'Matrilineal population structure and distribution of the Angolan giraffe in the Namib desert and beyond', Ecological Genetics and Genomics 7-8, 1-5. https://doi.org/10.1016/j.egg 2018.03.003 